\title{
Hydrothermal simulations in Brazil using batteries - First Results
}

\author{
C. Dall’Orto ${ }^{1}$, B. Bezerra ${ }^{1}$, R. Novaes ${ }^{1}$, F. Nazaré ${ }^{1}$, P. Rosas², P. Furlanetto ${ }^{3}$, W. Teixeira ${ }^{4}$, J. Tuo ${ }^{5}$, C. \\ Xinjian $^{5}$, Chai Jiyong 5 \\ ${ }^{1} \mathrm{PSR}$ \\ Praia de Botafogo 228 Sala 1701A \\ Botafogo - Rio de Janeiro, 22250-145 Rio de Janeiro (Brazil) \\ Phone/Faxnumber:+0055 19 39062100, e-mail: celso@psr-inc.com \\ ${ }^{2}$ Universidade Federal de Pernambuco(UFPE) \\ Av. Prof. Moraes Rego, 1235 - Cidade Universitária, Recife - PE - CEP: 50670-901 \\ Fone PABX: +55 (81) 2126.8000, pedro.rosas@ufpe.br \\ ${ }^{3}$ Instituto de Tecnologia Edson Mororo Moura- ITEMM \\ Rua José Vieira de Souza, 275 - Edson Mororo Moura, Belo Jardim- PE, 55150-550 \\ Phone:+55 (81) 3411 4111, paola.furlanetto@itemm.org.br \\ ${ }^{4}$ CPFL Energia (CPFL) \\ Rod. Eng. Miguel Noel Nascentes Burnier, 1755- Chácara Primavera, Campinas - SP, 13088-900 \\ Fone PABX:+55 (19) 3756 8517, wendell@cpfl.com.br \\ ${ }^{5}$ State Grid international Development Co. Ltd \\ Rod. Eng. Miguel Noel Nascentes Burnier, 1755 - Chácara Primavera, Campinas - SP, 13088-900 \\ Fone PABX: +55 (19) 3756 8517, jituo@cpfl.com.br
}

\begin{abstract}
The share of renewable energy sources has increased worldwide, especially those considered unconventional (i.e. without considering hydropower). Besides proved as economically competitive, these sources cause less environmental impacts when compared to other sources of traditional power generation such as coal and oil. However, solar and wind power plants are intermittent, i.e., the generation varies according to the availability of the respective natural resources and therefore are considered non-dispatchable by the System Operator (SO). Because of that, the electrical systems must be able to somehow compensate the variability from the non-dispatchable energy sources to meet the supply-demand equilibrium. Storage technologies such as electrochemical (e.g. batteries) and electromechanical (e.g. flywheel) could be used in that sense. Recent developments of these technologies opened space for several ancillary services and products to be offered by the storage systems to the electrical system. In addition to that, the sustained reduction in operation and maintenance (O\&M) costs summed to the payments for providing ancillary services could make some of these technologies economically attractive, under certain conditions. Given this new scenario, the representation of these storage systems in the expansion planning and power systems mathematical models becomes necessary. In this study, we simulated the Brazilian electrical system at 2035, considering batteries (corresponding to $7 \%$ of the installed capacity) and a $30 \%$ share of unconventional renewable energy in the system generation capacity.
\end{abstract}

\section{Key words}

Hydrothermal Dispatch, Energy Storage System, Batteries, Brazilian Electrical Power System

\section{Introduction}

The steady growth in the share of renewable energy sources in the world, influenced mainly by the concems over the greenhouse gases emission, has altered the mix of energy sources in several countries. The main renewable sources considered unconventional, that are not able to store energy as the hydroelectric plants, are the solar and wind power plants. These plants outputs are intermittent, i.e., the generation varies according to the availability of the natural res ource and, therefore, are als o considered as non-dispatchable by the SO.

This change in the mix has brought new challenges in the regulation, planning and operation of the electricity sector, mainly due to the large generation variations in short time frames. In countries with high shares of hydropower, traditionally, the hydroelectric plants with res ervoirs have been used to compensate the variability fromother energy sources and provide operating reserve to meet the offer demand equilibrium. However, environmental constraints and political pressures, coupled with the concerns about 
climate changes, dis couraged the planning and construction of new hydro power plants with large reservoirs. Therefore, recent research has focused on the development of other storage technologies, such as electrochemical and electromechanical.

From a planning point of view, a new mix of renewable energy sources increase the concerns about supply security, reliability and energy balance. From an operational perspective, a decentralized operation with unpredictable and not dispatchable large amounts of power, due to the participation of prosumers, presents challenges in tems of stability and quality of electricity supply.

Foreseeing the need for storage capacity in the electrical systems, the entire production chain has been preparing for the dissemination of battery-based storage systems (the socalled Battery Energy Storage Systems, or BESS). There is a broad consensus that this type of system has a very significant potential and will be a key is sue for the future of the electricity sector. The two main factors contributing to this conclusion are (i) the downward trend in battery prices over the past few years, driven by incremental technological gains and by the increasing demand for applications - e.g., portable electronics and electric vehicles [1]; and (ii) the increasing the need for ancillary services that could be provided by BESS.

The energy storage technology has been characterized as being still uncertain, but with great potential impact in the electricity sector by the World Energy Council [2], as illustrated in Figure 1.

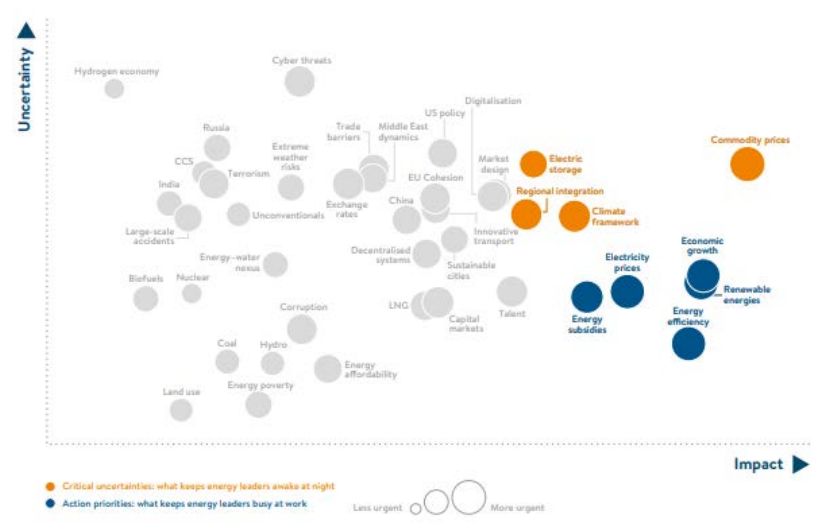

Figure 1 - Energy storage impacts on the electricity sector

Storage systems can provide a broad range of services, in part because of the great diversity of developing technologies, as discussed in the next section.

\section{Storage technologies}

Overall, there are many storage technologies available and at different levels of maturity, ranging from research and development to commercial applications already quite developed. In general, these technologies convert electrical energy during the charging phase into another form of storable energy, such as chemical, mechanical or thermal energy. In the discharge process the previously stored energy is again transformed into electricity. It is noteworthy that in all technologies there is a loss of energy in the charge-discharge process, and this is what determines the storage systemefficiency.
A widely known approach to classify storage systems is according to the way energy is used. For example, in Figure 2, systems are classified into electrochemical, electromechanical, hydro reversible, heat and hydrogen cell, according to the DOE database [3].
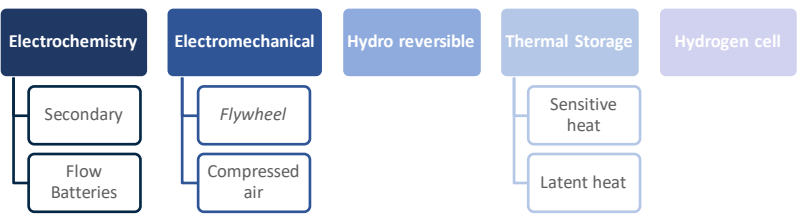

Figure 2 - Batteries technologies

With respect to batteries, the technologies used in the various forms of storage also varies, influencing the lifetime, the storing capacity, the charge cycle, and so on. In this way, various services can be provided, and for various sectors of the energy market. Some of these services will be discussed in Section 3 .

\section{Services provided by batteries}

The main services demanded by the electrical system are related to the supply-demand balance and are largely linked to the energetic services of a classic energy market, which involves energy transfers across the systemaiming to increase the stored energy as to hedge the systemagainst most critical supply situations. Although different applications may have focused on different aspects of the demand curve (e.g. the solar generation profile, the consuming profile at peak hours and at night, wind power spillages, etc.) generally these services have the common function of stabilizing the demand curve for all times at all grid points. In this case, the BESS can be paid largely by the energy spot market, in an hourly pricing environment. Note, however, that there is still room for regulatory improvements when evaluating the BESS contributions to the supply-demand balance. Notably, BESS can reduce system peak demand, also reducing the need for capacity contracting to maintain a margin of secure operative reserve: however, this contribution to the system secunity is still not properly remunerated in many countries.

Ancillary services represent contributions to the stability of the system, allowing it to be operated robustly, making it possible to compensate variations on delivered power. Among the most discussed ancillary services one can find voltage regulation, frequency regulation, black start capability and operating reserves. Such services can, in principle, be provided by any agent connected to the transmis sion network and are associated with the ability to change the power injections to increase the network stability - usually they are provided by large facilities. It is worthy to be mentioned that many countries lack some explicit compensation scheme for the provision of ancillary services, as the generators that provide such services may be indirectly remunerated. More modem pricing mechanisms are being studied in literature, such as the determination of a spot price for ancillary services, obtained from the co-optimization energy dispatch and operating res erve allocation requirements. 
A third category of services is associated with the system resilience and autonomy. A resilient, reliable, autonomous system can deliver electricity in quantity and quality demanded by consumers and is able to rapidly recover from an extreme event, such as the outage of a generator. Largely, these applications are associated with isolated systems, which cannot rely on the inertia of large networks to ensure stability during transient events. Another application is the one related to continuity of supply under load curtailment events and to the quality of service (voltage and current) for more sensitive applications. Either cases, typically these services are remunerated by apportionment between its beneficiaries.

Finally, there is support to transmission and distribution networks, more directly associated with the expansion and maintenance activities. These functions involve the BESS contributions that primarily reduce the cost of transmission services, such as management of power congestions, improvements in the distribution and transmis sion services quality and postponement of investments. One concem at this point is the need to have investments validated by regulators in the case of innovative technologies like the BESS - a risk for the electricity companies.

Summarizing this prelude, the BESS could be used in various applications: assist the system as a whole (e.g. acting in frequency control, meeting operating reserves, reducing transmis sion congestions) or some of its regions such as wind or solar power plants in order to increase the amount energy supplied to the system (e.g. avoiding spillages), increasing the supplied power quality (increasing the times when the parks can provide energy) and reducing the volatility of generation. Storage systems can als o bring relevant economic benefits by increasing efficiency and us eful life of assets, for example allowing a more immediate power compensation in the network.

When it comes to energy storage solutions in the current context, it generally points to the use of lithium ion batteries. This type of battery has gained much space in the market as it is highly adapted to implementations in distributed services technology, enabling the provision of different services to the network and the use of synergies. Another factor is the reduction in the investment cost in recent years, and the expectation of continued reduction, as illustrated in Figure 3, originally presented in [4].

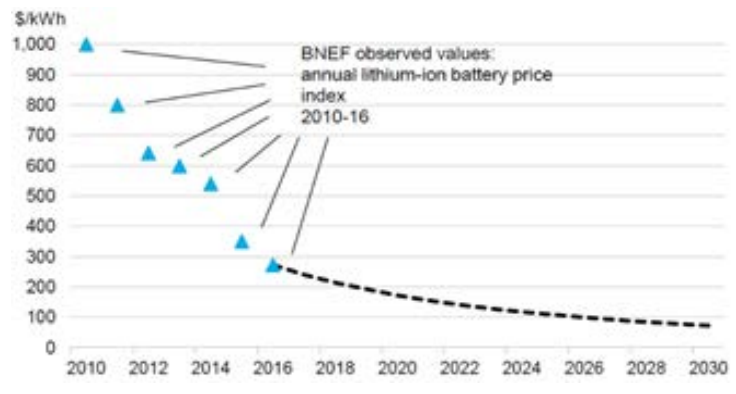

Figure 3 - Projection of cost reduction for the lithium ion batteries

Thus, it becomes mandatory to consider the energy storage systems (in addition to the hydroelectric res ervoirs) in the models of expansion and operation of electrical systems.

\section{Modelling batteries in dispatch model}

In Brazil, as in many other countries, thedecision-making related to dispatch decisions is based on computer optimization models. Thesemodels minimize the expected value of operating cost over a period of study, given a set of technical constraints and under uncertainties related to the availability of primary energy (e.g., wind, natural inflows), fuel costs and energy demand. Operating costs are mainly composed by fuel costs of thermal plants and possible energy shortages.

Despite the many services that batteries are able to provide, the goal here was to analyse the temporal energy transfers at different energy prices, where the energy generated under low marginal costs is stored to be used when the prices are higher. Thus, the batteries were simulated considering the technical characteristics of the storage system, such as loading and unloading curve, maximum power injection, energy balance, costs, among others.

This study employed the stochastic dual dynamic programming (SDDP) method $[5,6]$, using the SDDP ${ }^{\circledR}$ software, developed by PSR ${ }^{1}$. In addition to all the constraints already widely known in literature for the hydrothermal dispatch problem, new ones were included in the model in order to adequately represent thebatteries. The maximum battery discharge is represented by Eq.1.

$$
\boldsymbol{d}_{b, k} \leq \overline{\boldsymbol{p}}_{\boldsymbol{b}} \quad \forall \boldsymbol{b} \in \boldsymbol{B} ; \forall \in \boldsymbol{K}
$$

where:

$\boldsymbol{b}$ are the batteries indexes

$\boldsymbol{B}$ is the set of batteries

$\boldsymbol{k}$ are the demand levels indexes

$\boldsymbol{K}$ is the set of demand levels

$\boldsymbol{d}_{\boldsymbol{b}, \boldsymbol{k}}$ is the discharge of battery $\boldsymbol{b}$ in block $k$ [MWh]

$\overline{\boldsymbol{p}}_{\boldsymbol{b}}$ is the maximum discharge capacity of battery $\boldsymbol{b}$ [MWh]

The equation below represents the maximum battery charge:

$c_{b, k} \leq \bar{p}_{b} \quad \forall \boldsymbol{b} \in B ; \forall \boldsymbol{k} \in \boldsymbol{K}$

where:

$\boldsymbol{c}_{\boldsymbol{b}, \boldsymbol{k}}$ is the charge of battery $b$ in block $k$ [MWh]

The energy balance is written for all the batteries in Eq.3.

$$
v_{b, t+1}=v_{b, t}+\sum_{k \in K}\left[\beta_{b} c_{b, k}-d_{b, k}\right] \quad \forall b \in B
$$

where:

$\boldsymbol{t}$ are the time stage indexes

$\boldsymbol{v}_{\boldsymbol{b}, \boldsymbol{t}}$ is the stored energy in battery $\boldsymbol{b}$ at the end of stage $\boldsymbol{t}$ [MWh]

$\boldsymbol{\beta}_{\boldsymbol{b}}$ is the charge efficiency of battery $\boldsymbol{b}$ [p.u.]

The batteries' storage limits (maximumand minimum) are represented by Eq.4 and Eq.5, respectively.

\footnotetext{
${ }^{1}$ https://www.psr-inc.com/en/.
} 


$$
v_{b, t+1} \leq \bar{v}_{b} \quad \forall \boldsymbol{b} \in \boldsymbol{B}
$$

where:

$\bar{v}_{b}$ is the maximum storage of battery $b$ [MWh]

$$
v_{b, t+1} \geq \underline{v}_{b} \quad \forall \boldsymbol{b} \in \boldsymbol{B}
$$

where:

$\underline{v}_{b}$ is the minimum storage of battery $b[\mathrm{MWh}]$

Eq.6 guarantees that the energy demand will be met by supply in all stages and demand levels.

$$
\sum_{i \in I} g_{i, t, k}+\sum_{j \in J} g_{j, t, k}+\sum_{b \in B}\left[\gamma_{b} d_{b, k}-c_{b, k}\right]=d_{t, k} \quad \forall k \in K
$$

where:

$\boldsymbol{i}$ are the hydro generators indexes

$I$ is the set of hydro generators

$\boldsymbol{j}$ are the thermal generators indexes

$J$ is the set of thermal generators

$\boldsymbol{g}_{\boldsymbol{i}, \boldsymbol{k}, \boldsymbol{t}}$ is the output of the $\boldsymbol{i}_{\boldsymbol{t} \boldsymbol{h}}$ hydro generator at level $\boldsymbol{k}$ and stage $t$

$\boldsymbol{g}_{\boldsymbol{j}, \boldsymbol{k}, \boldsymbol{t}}$ is the generation of the $\boldsymbol{j}_{\boldsymbol{t} \boldsymbol{h}}$ thermal generator at level $\boldsymbol{k}$ and stage $\boldsymbol{t}$

$\boldsymbol{\gamma}_{\boldsymbol{b}}$ is the discharge efficiency of battery $\boldsymbol{b}$ [p.u.]

$\boldsymbol{d}_{\boldsymbol{k}, \boldsymbol{t}}$ is the energy demand at level $\boldsymbol{k}$, stage $\boldsymbol{t}$ [MWh]

\section{Study case}

The Brazilian electrical system is is divided between the generation, transmission, distribution, supply, market operation, import and export. The Brazilian system is widely known for being a hydrothermal system (hydropower plants account for 68\% of the generating capacity) with significant storage capacity in its reservoirs. Accordingly to data retrieved from the Brazilian System Operator website - ONS ${ }^{2}$, currently this power system has almost 160 GW of installed capacity and an average yearly demand of $66 \mathrm{GW}$. The peak demand verified in the country in 2017 was 83 GW. The share of thermal plants in the generation capacity is nowadays up to $22 \%$. Nonconventional renewable sources (i.e., solar and wind), however, have gained space in the market in the past decade ( $8 \%$ and $1 \%$ of actual installed capacity, respectively). In this regard, some support mechanisms for renewable sources have been offered by the Brazilian government, however there isn't yet a regulatory framework that provides support or encouragement to storage solutions, although the Brazilian regulator considers storage technologies in general as beneficial to the country and states that it could be used within the scope of ancillary services. The structure of the electricity

The hourly simulation was carried out for 2035, adopting PSR' supply and demand expansion assumptions ${ }^{3}$. The

${ }^{2}$ Information only available in Portuguese at http://ons.org.br.

${ }^{3}$ This scenario was constructed using the Optgen expansion planning model, developed by PSR, using data resulting supply mix is mostly composedby hydroelectric and unconventional renewable sources, with a reduced thermal share. To analyse the effect of the batteries, a second simulation was performed, in which three $6 \mathrm{GW}$ batteries were inserted, each of which had a maximum storage of $24 \mathrm{GWh}$ and a 0.85 p.u. global efficiency factor. The resulting supply mix can be found in Figure 4.

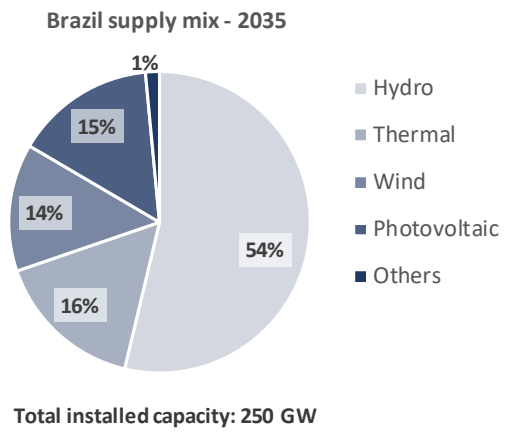

Figure 4 - Brazilian supply mix in 2035

\section{Results}

Based on supply and demand setting designed for 2035, the hydrothermal dispatch was simulated over the entire horizon using the stochastic optimization model SDDP, developed by PSR. The simulation was performed with hourly steps and considering uncertainties in inflows to hydroelectric plants, wind power and solar production.

Figure 5 shows the average generation by source and the spot price for the month of March. On might notice that spot prices follow the demand profile in Brazil, where it's necessary to dispatch natural gas thermoelectric plants to meet the peak demand. The solar production pattern is shown by the lightest blue area on the graph. The darkest blue area shows the operation of 18 GW of batteries, charging overnight and unloading during the day.

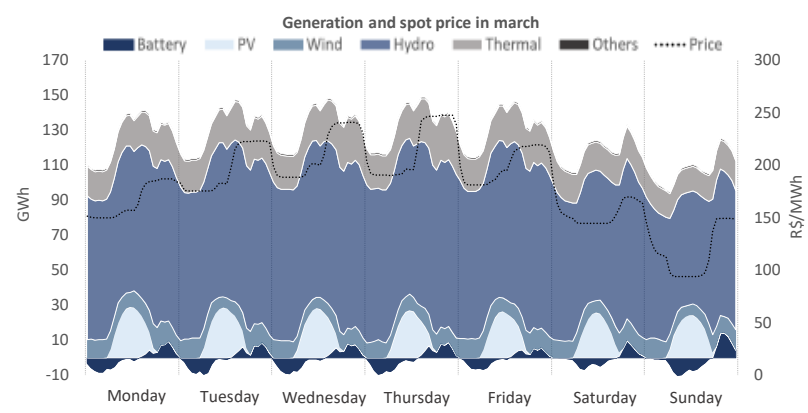

Figure 5 - Weekly generation profiles and spot prices in March

Figure 6 shows the generation profiles for the month of August, dry-season in Brazil. Note the decrease in the hydroelectric participation in the generation and the increase in the generation called as "Others", mostly composed by thermal plants fuelled with sugar cane bagasse, known for being complementary to the hydro source. Also, this profile shows that during summer

from the Brazilian Energy Res earch Office - EPE, available at http://epe.gov.br/en. We won't go further about this expansion plan, as it isn't in the scope of the present work. 
batteries are more commonly required to operate- in March batteries delivered 5.1 GW of power when dis charging.

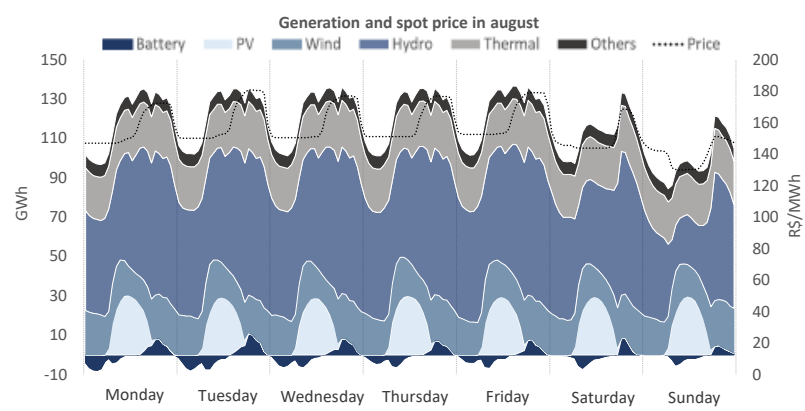

Figure 6 - Weekly generation profiles and spot prices in August

Figure 7 compares the operation of the batteries with the spot price of the system for the month of January. It is possible to see how the charge-discharge cycles follows the system's marginal cost profile.

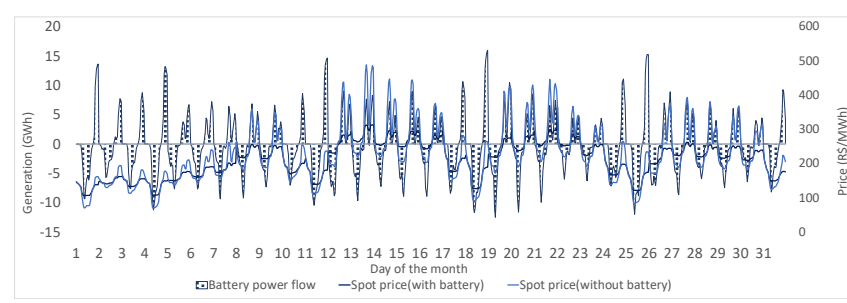

Figure 7 - Battery generation and spot prices in January

The figure als o compares the spot prices obtained from the simulations with and without batteries. It is observed that during the summer the batteries help reduce spot prices during the system's peak hours, moving energy available in the morning to the hour of maximum demand of the day i.e, prices are usually decreasing, when the batteries are discharging, and increasing when batteries are charging. Based on the operation of the batteries and the spot system prices, income was calculated in the spot market for two scenarios: (i) scenario without batteries, accounting for the hypothetical income of the first entrants in the Brazilian systemand (ii) inserting $18 \mathrm{GW}$ of batteries. It is observed that scenario (ii) the expected revenue for 2035 is $\mathrm{R} \$ 4.52$ per $\mathrm{kW}$. This value is much lower than the annualized cost of a large battery system, estimated at about $\mathrm{R} \$ 350$ per $\mathrm{kW}$ of CAPEX. Even if the battery services are evaluated using the prices obtained for case (i), without batteries (which has considerably higher prices for some hours), the expected revenue would be $\mathrm{R} \$ 63.07$ per $\mathrm{kW}$ in 2035. This means that the economic viability of the batteries strongly depends on the sale of ancillary services (e.g., frequency regulation) or on government incentives and targeted policies. The presented results also illustrate the downside effect for the battery business model in the event of a massive penetration of this technology, which mitigates the time variability of spot prices, reducing the financial attractiveness.

\section{Conclusion}

The share of renewable energy res ources, mainly wind and solar, has increased a lot worldwide. These sources have the characteristic of being intermittent, which requires the system to provide other res ources able to deal with the rapid changes of generation level. This service, as well as others, could be provided by energy storage systems. With the increase of these systems, it is necessary to represent them in planning and operation optimization models of electrical sys tems, in order to obtain coherent results. This representation, together with appropriate regulation, locational prices and greater discretization could make BESS economically viable. This paper presents a case study that shows the benefits of batteries in the marginal cost reduction in system peak and the attenuation of the time variability of the spot prices in the Brazilian system. Results show that batteries are not yet financially attractive. The reduction in the cost of BESS, and increased equipment life, can bring benefits for planning and operation of the system, for generators and consumers.

\section{Acknowledgement}

The developments presented in this article come from the ANEEL Strategic R\&D project "PA3026 - Integration of Storage Systems in Multiple Configurations to Suppoit Wind Generation", developed by PSR, ITEMM - Edson Mororo Moura Institute of Technology and UFPE Federal University of Pernambuco, financed by CPFL Paulista, CPFL Piratininga, Paulis ta Lageado Energia S/A, RGE Sul, CPFL Transmission Piracicaba SA, CPFL Centrais Geradoras LTDA, EPASA, Foz do Chapecó Energia SA and CPFL Renováveis.

We thank ANEEL, the technicians of all the companies involved and the CPFL group.

\section{References}

[1] MIT Energy Initiative. Utility of the fut ure: An MIT Energy Initiative response to an industry in transition. MIT, Cambridge, MA, 2016.

[2] World Energy Issues Monitor 2017 World Energy Council. Available in:https://www.worldenergy.org/wpcontent/uploads/2017/04/1.-World-Energy-Issues-Monitor-

2017-Full-Report.pdf, Accessed June 2018.

[3] DOE Global Energy Storage Database: Office of Electricity Delivery \& Energy Reliability. Available: http://www.energystorageexchange.org

[4] Bloomberg New Energy Finance. 2017. New Energy Outlook.

[5] Pereira, M.V.F., Optimal st ochastic scheduling operations of large hydroelectric systems. International Journal of Electrical Power \& Energy Systems, Kidlington, v.11, n.3, p.161-169, July 1989.

[6] Pereira, M.V.F., Pinto, L.M.G.V, St ochastic optimization of the multireservoir hydroelectric system: a decomposition approach. Water Resources Research, Washington, DC, v.21, n.6, p.779-792, jun 1985.

[7] Asif A.A, Singh R., Further Cost Reduction of Battery Manufacturing. MDPI, Batteries, 2017.

[8] SP Burger, M Luke. Business models for distributed energy resources: A review and empirical analysis. Energy Policy, Elsevier, 2017.

[9] United States Department of Energy. Donate Global Energy Storage Database2016 Database.

Available: http://www.energystorageexchange.org, Accessed June 2018. 